\title{
CAPTURE OF IRREGULAR SATELLITES AT JUPITER
}

\author{
David Nesvorn ${ }^{1}$, David VoKrouhlickÝ ${ }^{1,2}$, AND Rogerio DeIENNO ${ }^{1,3}$ \\ ${ }^{1}$ Department of Space Studies, Southwest Research Institute, 1050 Walnut Street, Suite 300, Boulder, CO 80302, USA \\ ${ }^{2}$ Institute of Astronomy, Charles University, V Holešovičkách 2, 18000 Prague 8, Czech Republic \\ ${ }^{3}$ Division of Space Mechanics and Control, National Institute of Space Research, São José dos Campos, SP 12227-010, Brazil \\ Received 2013 November 1; accepted 2014 January 27; published 2014 February 27
}

\begin{abstract}
The irregular satellites of outer planets are thought to have been captured from heliocentric orbits. The exact nature of the capture process, however, remains uncertain. We examine the possibility that irregular satellites were captured from the planetesimal disk during the early solar system instability when encounters between the outer planets occurred. Nesvorný et al. already showed that the irregular satellites of Saturn, Uranus, and Neptune were plausibly captured during planetary encounters. Here we find that the current instability models present favorable conditions for capture of irregular satellites at Jupiter as well, mainly because Jupiter undergoes a phase of close encounters with an ice giant. We show that the orbital distribution of bodies captured during planetary encounters provides a good match to the observed distribution of irregular satellites at Jupiter. The capture efficiency for each particle in the original transplanetary disk is found to be $(1.3-3.6) \times 10^{-8}$. This is roughly enough to explain the observed population of jovian irregular moons. We also confirm Nesvorný et al.'s results for the irregular satellites of Saturn, Uranus, and Neptune.
\end{abstract}

Key word: planets and satellites: formation

Online-only material: color figures

\section{INTRODUCTION}

The four outer planets in the solar system have a significant population of satellites with large, elongated, inclined, and often retrograde orbits (see, e.g., Nicholson et al. 2008 for a review). These irregular satellites are thought to have been captured by planets from heliocentric orbits, because the circumplanetary disk processes that are thought to be responsible for the formation of the regular satellites are not expected to lead to the orbital characteristics of the irregular satellites. Many capture mechanisms have been proposed in the past (e.g., Colombo \& Franklin 1971; Heppenheimer \& Porco 1977; Pollack et al. 1979; Ćuk \& Burns 2004; Philpott et al. 2010; Suetsugu $\&$ Ohtsuki 2013). Here we consider the capture mechanism suggested in Nesvorný et al. (2007, hereafter NVM07).

NVM07 proposed that the irregular satellites were captured around giant planets when the orbital instability in the outer solar system triggered a phase of close encounters of Uranus and Neptune to Jupiter and Saturn. Each encounter involves a complex gravitational interaction of a gas giant, an ice giant, and numerous background planetesimals. If the encounter geometry is right, the trajectory of a background planetesimal can be influenced in such a way that the planetesimal ends up on a bound orbit around one of the planets, where it remains permanently trapped when planets move away from each other.

To illustrate this concept, NVM07 used the model of planetary instability and migration proposed by Tsiganis et al. (2005; hereafter the original Nice model or ONM for short). In the ONM, the outer planets were placed between 5 and 18 AU. The instability was triggered when Jupiter and Saturn migrated over their mutual 2:1 resonance. Following this event, the orbits of Uranus and Neptune became Saturn-crossing, Uranus and Neptune were scattered out by Saturn, and these planets stabilized and migrated to their current locations by gravitationally interacting with an outer planetesimal disk.

The encounters between planets in the ONM remove distant satellites that may have initially formed at Saturn, Uranus, and
Neptune by gas-assisted capture (or via a different mechanism). A new generation of satellites, however, can be captured from the background planetesimal disk during planetary encounters. By modeling this process, NVM07 showed that the capture efficiency and orbital distribution of bodies captured at Saturn, Uranus, and Neptune are consistent with observations.

Jupiter, however, does not generally participate in planetary encounters in the ONM. This is a consequence of the choice of the initial conditions in the ONM (Tsiganis et al. 2005), where an ice giant scattered off of Saturn is subsequently stabilized in the outer disk (without ever evolving onto the Jupiter-crossing orbit). The mechanism proposed in NVM07 was therefore not expected to produce the irregular satellites of Jupiter. Yet, the population of irregular satellites at Jupiter does not seem to be notably different from those at Saturn, Uranus, or Neptune (Jewitt \& Haghighipour 2007).

A new model of the dynamical instability in the outer solar system has been recently developed to address several shortcomings of the ONM. This model, known as jumping Jupiter, was proposed to avoid problems with excessive excitation of the terrestrial planets that would occur if Jupiter and Saturn slowly migrated past the 2:1 mean motion resonance, or MMR (Brasser et al. 2009, 2013; Agnor \& Lin 2012), to explain the dynamical structure of the asteroid belt (Minton \& Malhotra 2009; Morbidelli et al. 2010), and to obtain a correct distribution of secular modes for Jupiter and Saturn (Morbidelli et al. 2009a).

The initial orbits of planets in the jumping-Jupiter model were chosen to respect our expectations for the previous stage when planets formed and migrated in the protoplanetary gas disk. Jupiter and Saturn, for example, are expected to be in the 3:2 resonance at the time of the gas disk dispersal (e.g., Masset $\&$ Snellgrove 2001). The inner ice giant probably had a nearby resonant orbit as well. These orbital configurations are more compact than the ones considered in the ONM such that there is more opportunity for planetary encounters.

Of particular importance to this work is that, in the jumpingJupiter model, Jupiter undergoes a series of encounters with 
Uranus, Neptune, or the ejected ice giant (Nesvorný 2011; Batygin et al. 2012; Nesvorný \& Morbidelli 2012; hereafter NM12). This may resolve the problem with the NVM12 results discussed above, because the irregular satellites of Jupiter could have been captured during these encounters. Here we study the capture of irregular satellites at Jupiter in the instability models taken from NM12. The purpose of this paper is to understand whether by adding an ice-giant planet to the system, the origin of the irregular satellites of Jupiter can be explained assuming the same capture mechanism suggested in NVM07. Our methods are explained in Section 2. The capture efficiency and orbital distribution of captured bodies are reported in Section 3. Section 4 concludes this paper.

\section{CAPTURE SIMULATIONS}

Here we work with three cases taken from NM12. Their properties were illustrated in Nesvorný et al. (2013, their Figures 1-4). They were selected from NM12 as three representative instability simulations that satisfy the success criteria defined in NM12 (i.e., generate correct orbits of the outer planets, avoid excessive excitation of the inner planets, and produce correct amplitude of the proper eccentricity mode of Jupiter's orbit). In all three cases, the solar system was assumed to have five giant planets initially (Jupiter, Saturn, and three ice giants). This is because NM12 showed that having five planets initially is convenient to obtain jumping Jupiter and satisfy constraints. The third ice giant with the mass comparable to that of Uranus or Neptune is ejected into interstellar space during the instability (Figure 1; see also Nesvorný 2011; Batygin et al. 2012). A shared property of the selected runs is that Jupiter undergoes a series of encounters with the ejected ice giant.

NM12's simulations were performed using the symplectic integrator known as SyMBA (Duncan et al. 1998). The outer planetesimal disk in NM12 was represented by up to 10,000 particles distributed in an annulus with the inner edge at $r_{\text {in }}$ and the outer edge at $r_{\text {out }}$, where $r_{\text {in }}=20-24 \mathrm{AU}$, with the exact value depending on the specific case, and $r_{\text {out }}=30 \mathrm{AU}$. The surface density of particles in the annulus was set to $\Sigma \propto 1 / r$, where $r$ is the radial distance from the Sun. The eccentricities and inclinations of particles were set to be zero.

Because the capture probabilities are expected to be very low $\left(\sim 10^{-8}\right.$ for each disk particle; NVM07), the original number of disk particles in NM12 was largely insufficient to detect satellite capture directly. Instead, to be able to deal with this very low probability, we adopt the methodology developed in NVM07. This involves a three step process, where different numerical integrators are used to model the initial stage of the outer disk dispersal, planetesimal dynamics during the instability, and the capture process itself. At each transition, the results of the previous step are used to set up the initial conditions for the next step. The main goal of this procedure is to increase the statistics. $^{4}$

We start by characterizing the overall spatial density and orbital distribution of planetesimals as a function of time and location in the disk. This is done as follows. The selected NM12 runs are repeated with SyMBA, using the same initial conditions for planets and planetesimals, and recording the planetary orbits at $1 \mathrm{yr}$ time intervals. We then perform a second set of integrations with our modified version of the

\footnotetext{
4 With capture probabilities of the order $10^{-8}$, it would be impossible to carry out full-scale simulations of a very large number of disk particles that would result in reasonable statistics.
}

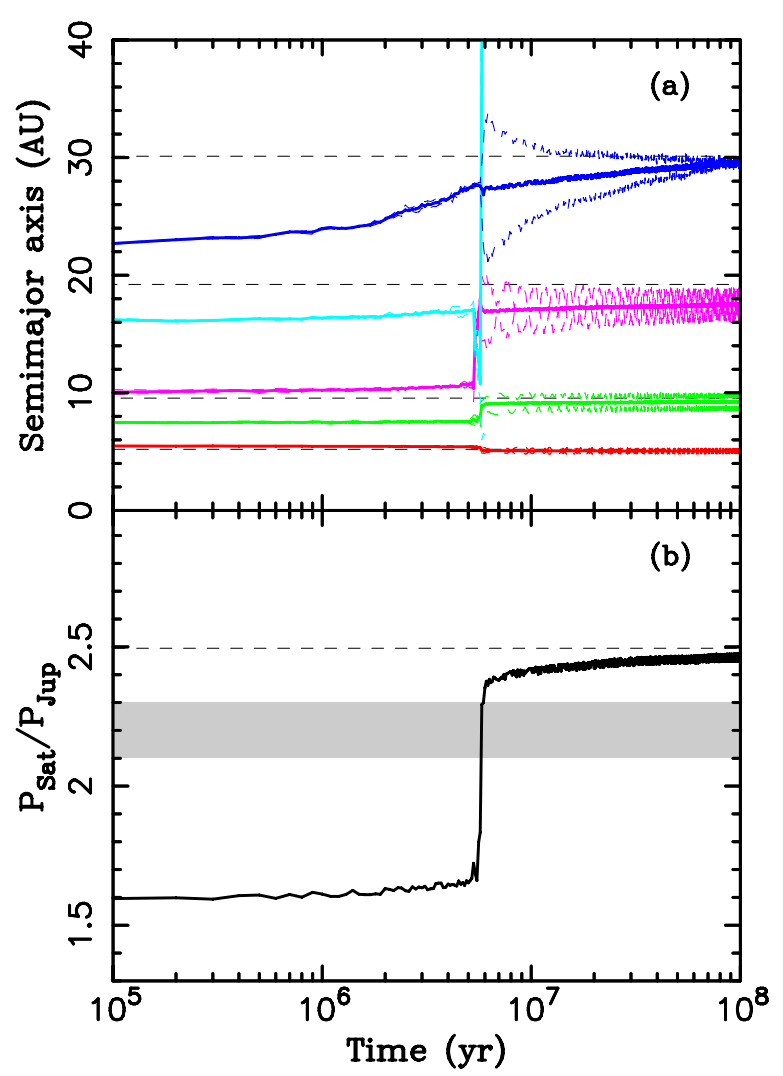

Figure 1. Orbital histories of the outer planets in Case 1. The planets were started in the $(3: 2,3: 2,2: 1,3: 2)$ resonant chain, and $M_{\text {disk }}=20 M_{\text {Earth }}$. (a) The semimajor axes (solid lines), and perihelion and aphelion distances (dashed lines) of each planet's orbit. The black dashed lines show the semimajor axes of planets in the present solar system. (b) The period ratio $P_{\text {Sat }} / P_{\mathrm{Jup}}$. The dashed line shows $P_{\mathrm{Sat}} / P_{\mathrm{Jup}}=2.49$, corresponding to the period ratio in the present solar system. The shaded area approximately denotes the zone where the secular resonances with the terrestrial planets occur.

(A color version of this figure is available in the online journal.)

swift_rmvs3 integrator (Levison \& Duncan 1994), where the planetary orbits are read from the file recorded by SyMBA and are interpolated to the required sub sampling (generally $0.25 \mathrm{yr}$, which is the integration time step used here). The interpolation is done in Cartesian coordinates (Nesvorný et al. 2013). ${ }^{5}$ This assures that the orbital evolution of planets in these new integrations is practically the same (up to small errors caused by the interpolation routine) as in the original SyMBA runs.

The swift_rmvs3 jobs were launched on different CPUs, with each CPU computing the orbital evolution of a large number of disk particles. The particles were considered massless so that they do not interfere with the interpolation routine (this is why swift_rmvs3, and not SyMBA, was used at this stage). The initial orbital distribution of each particle set was chosen to respect the initial distribution of disk particles in the original NM12 simulation, but differed in details (e.g., the initial mean longitudes of particles were random), so that each set behaved like an independent statistical sample. This allowed us to build

\footnotetext{
5 First, the planets are forward propagated on the ideal Keplerian orbits starting from the positions and velocities recorded by SyMBA at the beginning of each $1 \mathrm{yr}$ interval. Second, the SyMBA position and velocities at the end of each $1 \mathrm{yr}$ interval are propagated backward (again on the ideal Keplerian orbits). We then calculate a weighted mean of these two Keplerian trajectories for each planet so that progressively more (less) weight is given to the backward (forward) trajectory as time approaches the end of the $1 \mathrm{yr}$ interval. We verified that this interpolation method produces insignificant errors.
} 


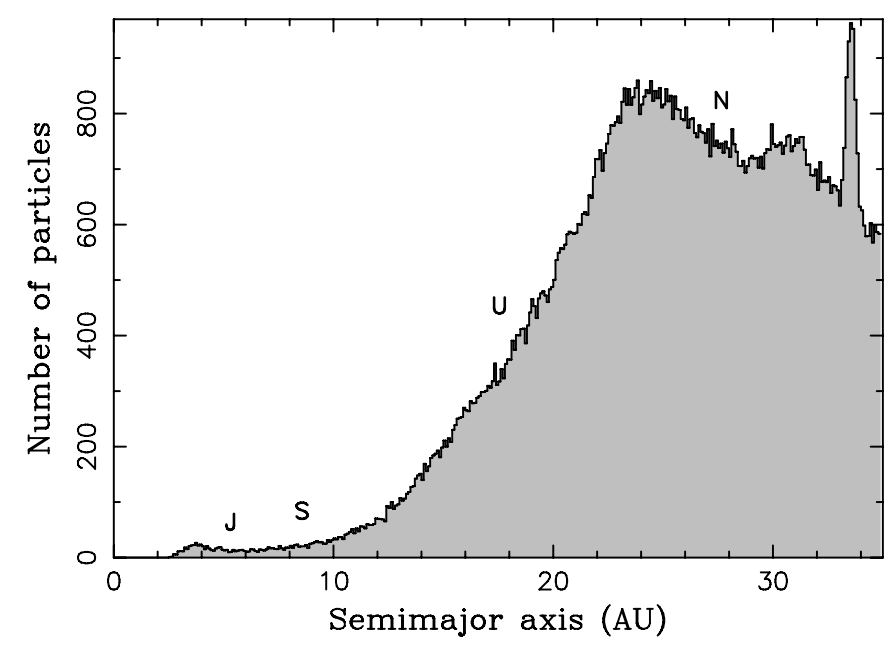

Figure 2. Semimajor axis distribution of disk particles in Case 1 at the time of the first encounter between Jupiter and an ice giant. The labels denote the semimajor axes of planets at this instant. The peak in the distribution at $\simeq 33.5 \mathrm{AU}$ corresponds to particles captured in the 3:2 MMR with Neptune.

up good statistics. In total, we followed $N_{\text {disk }}=300,000$ disk particles in each of the three selected cases. Figure 2 illustrates the semimajor axis distribution of disk particles in Case 1.

Our SyMBA integrations were also used to record all encounters between planets with encounter distance $r<R_{\mathrm{H}, 1}+R_{\mathrm{H}, 2}$, where $R_{\mathrm{H}, 1}$ and $R_{\mathrm{H}, 2}$ are the Hill radii of the two planets having an encounter. Then, in the swift_rmvs3 runs with the increased resolution $\left(N_{\text {disk }}=300,000\right)$, we drew a sphere with radius $3 \mathrm{AU}$ around each encounter and characterized the orbital distribution of disk particles in this "encounter zone". This was done individually for each encounter. In the following, the fraction of disk particles in the encounter zone will be denoted by $f_{3 \mathrm{AU}, i}=N_{3 \mathrm{AU}, i} / N_{\text {disk }}$, where $N_{3 \mathrm{AU}, i}$ is the number of disk particles in the zone and index $i$ runs over encounters. Typically, we find that $f_{3 \mathrm{AU}, i} \simeq 4 \times 10^{-4}$ for Jupiter.

In the final step, we used the Bulirsch-Stoer code that we adapted to this problem in NVM07, and followed the planets and disk particles through a sequence of encounters. The timing and geometries of planetary encounters were taken from the SyMBA integrations. For each encounter, the two planets were first integrated backward from the moment of their closest encounter until their separation increased to $3\left(R_{\mathrm{H}, 1}+R_{\mathrm{H}, 2}\right)$. We then included $N_{\text {test }}=4 \times 10^{7}$ test particles with the orbital distribution respecting that of the disk particles in the encounter zone. The mean longitudes of the particles were set such that, if particles were propagated on the strictly Keplerian heliocentric orbits, they would end up in the $3 \mathrm{AU}$ encounter zone at the time of the closest approach between planets. The forward integrations with planets and test particles were run up to $t=2 T$, where $t=T$ corresponds to the time of the closest approach.

After each planetary encounter, the Hill spheres of planets were searched for captured satellites. The orbits of the identified satellites were integrated to the next encounter (following the time sequence of encounters recorded by SyMBA). The unstable orbits were removed. The stable satellites were kept and included in the next encounter. By iterating the procedure over all encounters, we obtained the state of the satellite swarm at each planet after the last planetary encounter. We then followed the orbits of these satellites with a symplectic integrator for additional $100 \mathrm{Myr}$ to account for long-term instabilities and removal by collisions with large regular moons. ${ }^{6}$ It is not necessary to follow orbits over gigayear timescales, because the number of removed satellites at late stages is minimal. The final population of stable satellites is discussed below.

For consistency, we also evaluated the dynamical survival of regular moons during the planetary encounters in three cases considered here. The results of this part of the work will be reported in R. Deienno et al. (2014, in preparation).

\section{RESULTS}

Here we describe the results of the numerical integrations described above. The mechanism of capture is discussed in Section 3.1. We then examine the orbital distribution of captured irregular satellites and compare it with observations (Section 3.2). The capture probability and implications for the size distribution of planetesimals in the outer disk are discussed in Section 3.3. We focus on the irregular satellites of Jupiter, because they are the most troublesome case given that their capture does not occur in the ONM (NVM07). The irregular satellites of other planets are discussed in Section 3.4.

\subsection{Captured Satellites}

While the global evolution of planets was similar in the three selected cases (Nesvorný et al. 2013; their Figures 1-3), the history of Jupiter's encounters with an ice giant varied from case to case. These differences, which arise from slightly different initial conditions, can be important for satellite capture and is why different cases were considered in the first place (Figure 3). In Case 1, there were 59 encounters of Jupiter with an ice giant occurring over an interval of $200 \mathrm{kyr}$. Two of these encounters, one near the beginning and one near the end of the scattering phase, were very deep. In Case 2 , the scattering phase of Jupiter was richer, with 280 recorded encounters, and lasted over $300 \mathrm{kyr}$. In contrast, Case 3 showed a relatively poor history of Jupiter's encounters lasting 40 kyr only.

Figure 3 shows the number of satellites captured during different encounters at Jupiter. This plot illustrates, as already reported in NVM07, that satellites can be captured at nearly all close encounters. The satellites captured during early encounters, however, are often eliminated by the subsequent encounters. The satellites that survive subsequent encounters are typically the ones that were captured on tightly bound orbits (semimajor axis $a \lesssim 0.1 \mathrm{AU})$. The population of satellites builds up over time as more and more encounters contribute. The largest number of satellites was produced in Case 2, which is expected, because Case 2 has the largest number of encounters.

The satellites are captured in our simulations when the presence of the ice giant influences the initially hyperbolic fly-by of a test particle near Jupiter. Capture happens even during relatively distant encounters when the Hill spheres of the two planets barely overlap (Figure 3). In this case, particles passing near the ice giant cannot be captured on bound orbits about Jupiter, because they will enter Jupiter's Hill sphere on a hyperbolic trajectory. Instead, the gravity of the ice giant influences the trajectories of bodies passing deeply inside the Hill sphere of Jupiter, and changes them, if the encounter geometry is right, such that they become bound.

\footnotetext{
6 The large regular satellites are assumed to have formed at this stage. This should be obvious, because the regular satellites presumably formed in the circumplanetary disk within the first $\sim 10$ Myr after the condensation of first solar system solids, while here we are describing events that occurred much later.
} 

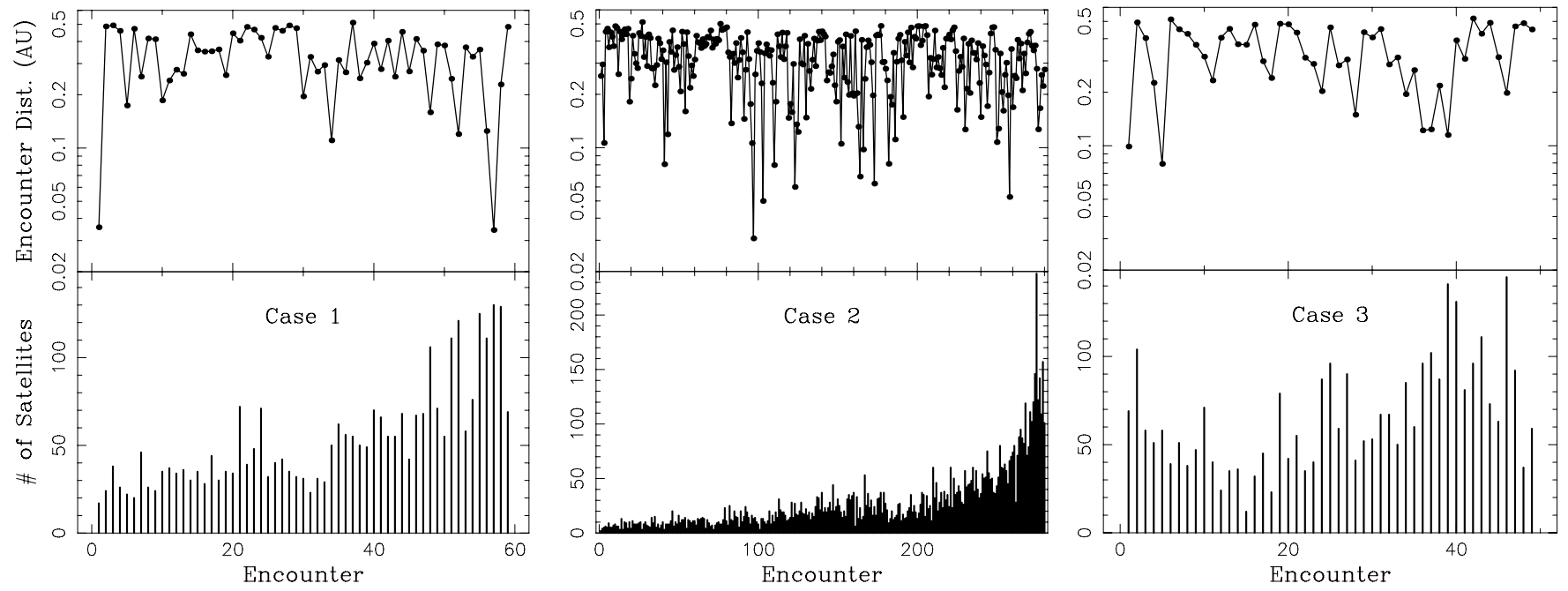

Figure 3. History of encounters (top) and number of satellites (bottom) captured at Jupiter in Cases 1, 2, and 3. Only the stable satellites, i.e., those that survive all subsequent encounters and 100 Myr past the encounter stage, are shown in the bottom histograms.
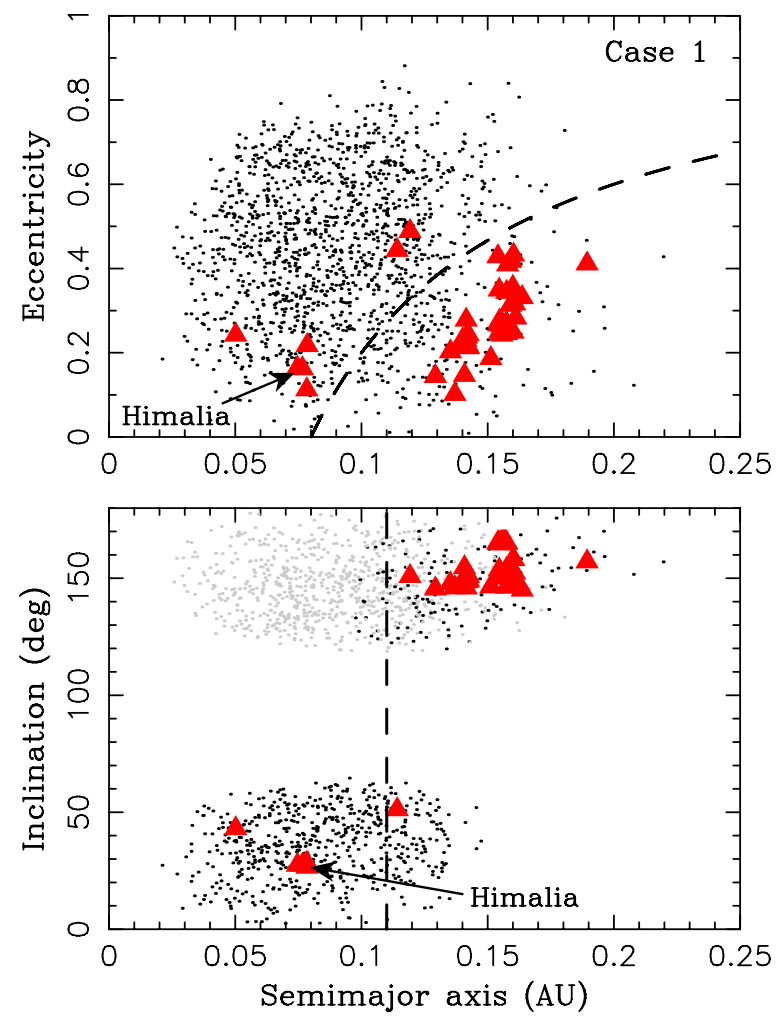

Figure 4. Orbits of satellites captured in Case 1 (dots) and known irregular satellites at Jupiter (red triangles). The dashed line in the top panel denotes $q=a(1-e)=0.08 \mathrm{AU}$, which is an approximate limit below which the population of small retrograde satellites becomes strongly depleted by collisions with Himalia (Nesvorný et al. 2003). The depleted orbits with $i>90^{\circ}$ and $q<0.08 \mathrm{AU}$ are shown by gray dots in the bottom panel. The dashed line in the bottom panel shows the boundary value $a=0.11 \mathrm{AU}$ below which the collisions with Himalia would remove more than $50 \%$ of small retrograde satellites (Nesvorný et al. 2003).

(A color version of this figure is available in the online journal.)

\subsection{Orbital Distribution}

The orbital distribution of satellites captured at Jupiter in Case 1 is shown in Figure 4. The orbital distributions obtained in Cases 2 and 3 are very similar to that of Case 1 and are not shown here. The semimajor axis of captured bodies ranges between 0.03 and $0.14 \mathrm{AU}$ for prograde orbits, and 0.03 and $0.2 \mathrm{AU}$ for retrograde orbits. The different outer extensions are dictated by different stability limits of the prograde and retrograde orbits (e.g., Nesvorný et al. 2003). The eccentricity distribution covers the whole range of values between 0 and $\simeq 0.7$. The orbits with $q=a(1-e)<0.015 \mathrm{AU}$ were removed, because of collisions with the Galilean satellites. The orbits with inclinations $60^{\circ}<i<120^{\circ}$ are strongly affected by the Kozai resonance (e.g., Carruba et al. 2002) and are removed as well once they reach $q<0.015 \mathrm{AU}$.

The comparison with orbits of known irregular satellites at Jupiter is satisfactory. The known prograde satellites fall well within the range of the model distribution. The distribution of known retrograde orbits is somewhat discordant with the model distribution in that all known retrograde satellites have $a>0.11 \mathrm{AU}$, while the semimajor axes of captured bodies extend down to $0.03 \mathrm{AU}$ (Figure 4, bottom panel). This can be understood, because the model distribution shown here does not account for collisions with the large prograde moon Himalia (mean radius $R \simeq 85 \mathrm{~km}$ ). Once these collisions are taken into account, it becomes apparent that the population of small retrograde satellites with $q<0.08 \mathrm{AU}$ must have been strongly depleted over gigayear timescales. For example, Nesvorný et al. (2003) estimated that most retrograde satellites with $a<0.11$ AU should be removed by collisions with Himalia.

We therefore conclude that the orbital distribution of Jovian irregular satellites was shaped in a rather intricate way by the capture process, long-term instabilities, collisions with the regular moons, and collisions of the irregular moons themselves (see also Bottke et al. 2010). The final orbital distributions are found to be relatively insensitive to the detail history of planetary encounters in the NM12 models, because all three cases considered here give similar orbital distributions. This result provides support to the NM12 models considered here, because these models were not selected with any a priori knowledge of what to expect.

\subsection{Capture Probability}

The probability of capture for each disk particle can be written as

$$
P_{\text {cap }}=\sum_{i=1}^{N_{\text {enc }}} f_{3 \mathrm{AU}, i} \frac{N_{\text {cap }, i}}{N_{\text {test }}}
$$




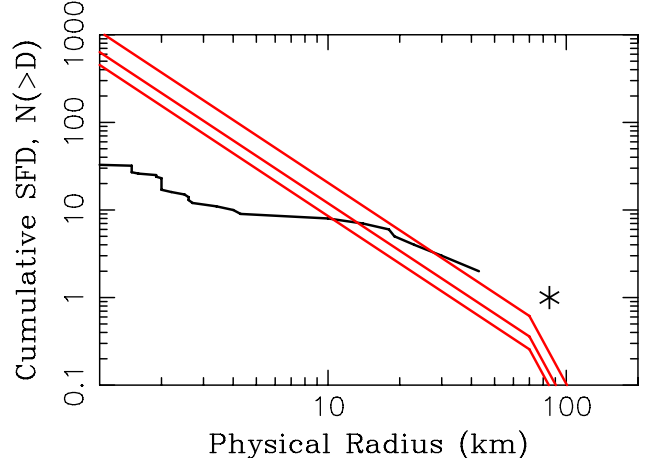

Figure 5. Cumulative size distribution of known irregular satellites at Jupiter (black line). The largest satellite, Himalia, is shown by an asterisk. The three red lines show the distributions expected from our model in Cases 2, 1, and 3 (from top to bottom).

(A color version of this figure is available in the online journal.)

Table 1

The Capture Statistics of Irregular Satellites at Jupiter

\begin{tabular}{lccc}
\hline \hline & Case 1 & Case 2 & Case 3 \\
\hline$N_{\text {enc }}$ & 59 & 280 & 49 \\
$f_{3 \mathrm{AU}}\left(10^{-4}\right)$ & 4.2 & 4.0 & 3.5 \\
$N_{\text {cap }}$ & 1458 & 3617 & 1403 \\
$P_{\text {cap }}\left(10^{-8}\right)$ & 1.5 & 3.6 & 1.3 \\
\hline
\end{tabular}

Note. The rows are: the (1) number of encounters $\left(N_{\mathrm{enc}}\right),(2)$ mean fraction of disk particles in the $3 \mathrm{AU}$ sphere around encounters $\left(f_{3 \mathrm{AU}}\right),(3)$ number of captured stable irregular satellites $\left(N_{\text {cap }}\right)$, and (4) probability of capture $\left(P_{\text {cap }}\right)$

where index $i$ goes over individual planetary encounters, $N_{\text {enc }}$ is the total number of recorded encounters, $f_{3 \mathrm{AU}, i}$ is the fraction of disk particles in the 3-AU-radius encounter sphere (see Section 2), $N_{\text {cap }, i}$ is the number of stable satellites captured during encounter $i$, and $N_{\text {test }}=4 \times 10^{7}$ is the number of test particles injected into the encounter sphere. The values of these parameters for our three cases are reported in Table 1 . We find that $P_{\text {cap }}=(1.3-3.6) \times 10^{-8}$. This is $1.5-4.2$ times more than $P_{\text {cap }}$ found in one isolated case in NVM07.

In Nesvorný et al. (2013), we used the very same three instability cases from NM12 considered here to study capture of Jupiter Trojans. The results implied, when calibrated from the observed population of Jupiter Trojans, that the outer planetesimal disk contained $\simeq(3-4) \times 10^{7}$ bodies with radius $R>40 \mathrm{~km}$. Using this calibration and the capture probability computed here, we find that roughly one irregular satellite with $R>40 \mathrm{~km}$ is expected to be captured at Jupiter. For comparison, two largest irregular moons, Himalia and Elara, have $R \sim 85 \mathrm{~km}$ and $R \sim 43 \mathrm{~km}$, respectively (the third largest is Pasiphae with estimated $R \sim 30 \mathrm{~km}$ ). This comparison is encouraging because it shows that the calculations are roughly consistent with the observed number of satellites.

Figure 5 shows the results in a plot. Here we assume that the shape of the size-frequency distribution (SFD) of Jupiter Trojans represents a good proxy for the SFD of planetesimals in the outer disk (Morbidelli et al. 2009b). If so, the SFD of disk planetesimals can be approximated by $N(>R)=N_{0}\left(R / R_{*}\right)^{-q_{1}}$ for $R>R_{*}=70 \mathrm{~km}$ and $N(>R)=N_{0}\left(R / R_{*}\right)^{-q_{2}}$ for $R<R_{*}$, where $q_{1} \simeq 5$ and $q_{2} \simeq 1.8$ (e.g., Fraser et al. 2014). The normalization constant $N_{0}$ is set such that $N(>40 \mathrm{~km}) \simeq$ (3-4) $\times 10^{7}$, as found in Nesvorný et al. (2013) by calibrating the SFD from Jupiter Trojans. We than convolve this SFD with the capture probability reported above.

The results shown in Figure 5 indicate that the capture process studied here is capable of capturing a much larger population of small irregular satellites than the currently known population of $R<10 \mathrm{~km}$ irregular moons of Jupiter. This can suggest one of several things. (1) The known population of small irregular moons is strongly incomplete. (2) The original population of small moons was much larger but was later removed by disruptive collisions (Nesvorný et al. 2003; Bottke et al. 2010). (3) The original SFD did not follow a simple power law below $10 \mathrm{~km}$. Instead, it was wavy.

Taken at its face value, the capture probability estimated here is somewhat inadequate to explain Jupiter's largest irregular moon, Himalia. This discrepancy becomes slightly smaller if the real dimensions of Himalia are $120 \mathrm{~km}$ by $150 \mathrm{~km}$, as reported by Porco et al. (2003), indicating effective $R \simeq 60-75 \mathrm{~km}$ (rather than $R \simeq 85 \mathrm{~km}$ taken from the JPL Horizons site and used in Figure 5). This would move Himalia closer to the model curves in Figure 5. Alternatively, Himalia may have been captured before the epoch of planetary encounters (e.g., Cuk $\&$ Burns 2004) and survived on a bound orbit. We find that the fraction of surviving jovian moons with $0.05 \mathrm{AU}<a<0.1 \mathrm{AU}$ is $\sim 0.01-0.3$ depending on the case considered (low in Case 2 and high in Cases 1 and 3 ).

We may have sub-estimated $P_{\text {cap }}$ by only considering encounters with $r<R_{\mathrm{H}, 1}+R_{\mathrm{H}, 2}$. In fact, as Figure 3 shows, even encounters with $r=R_{\mathrm{H}, 1}+R_{\mathrm{H}, 2} \simeq 0.5$ AU lead to capture of stable satellites. This raises the possibility that even encounters with $r>0.5 \mathrm{AU}$ can lead to satellite capture, which can be important, because the number of encounters increases with $r$ as $r^{2}$. Unfortunately, there is no easy way for us to include these distant encounters at this time, because this would essentially require to repeat the whole analysis. For reference, the original analysis required several months of computer time on $50 \mathrm{CPUs}$.

\subsection{Other Planets}

NVM07 showed that the populations of irregular satellites at Saturn, Uranus, and Neptune can be explained by capture during planetary encounters. Their model for planetary encounters, however, was based on the ONM (Tsiganis et al. 2005), such as it is not clear whether similar results can be obtained in the new instability cases considered here. We therefore discuss the results for these three planets below.

The orbital distributions obtained in all cases considered here represent a good match to observations (Figure 6). Relative to NVM07, we obtain a slightly broader semimajor axis range for satellites captured at Neptune (compare our distributions to those shown in Figures 4 and 5 in NVM07), which is good to explain the distant orbits of two known retrograde moons (Psamathe and Neso). We do not consider, however, this difference significant enough to discriminate between models. The orbital distributions of satellites captured at Saturn and Uranus are similar to those reported in NVM07.

As for the capture probability, here we obtain $P_{\text {cap }} \sim 5 \times 10^{-8}$ for Saturn and $P_{\text {cap }} \simeq(1-3) \times 10^{-8}$ for Uranus and Neptune. These values are a factor of several lower than those reported for Saturn, Uranus, and Neptune in NVM07. This should not be a problem, however, because the number of satellites captured at these planets in NVM07 were factor of several larger than needed. These differences are related to the detailed history of encounters in the instability models. With more encounters of Saturn, Uranus, and Neptune, as in NVM07, more satellites are 

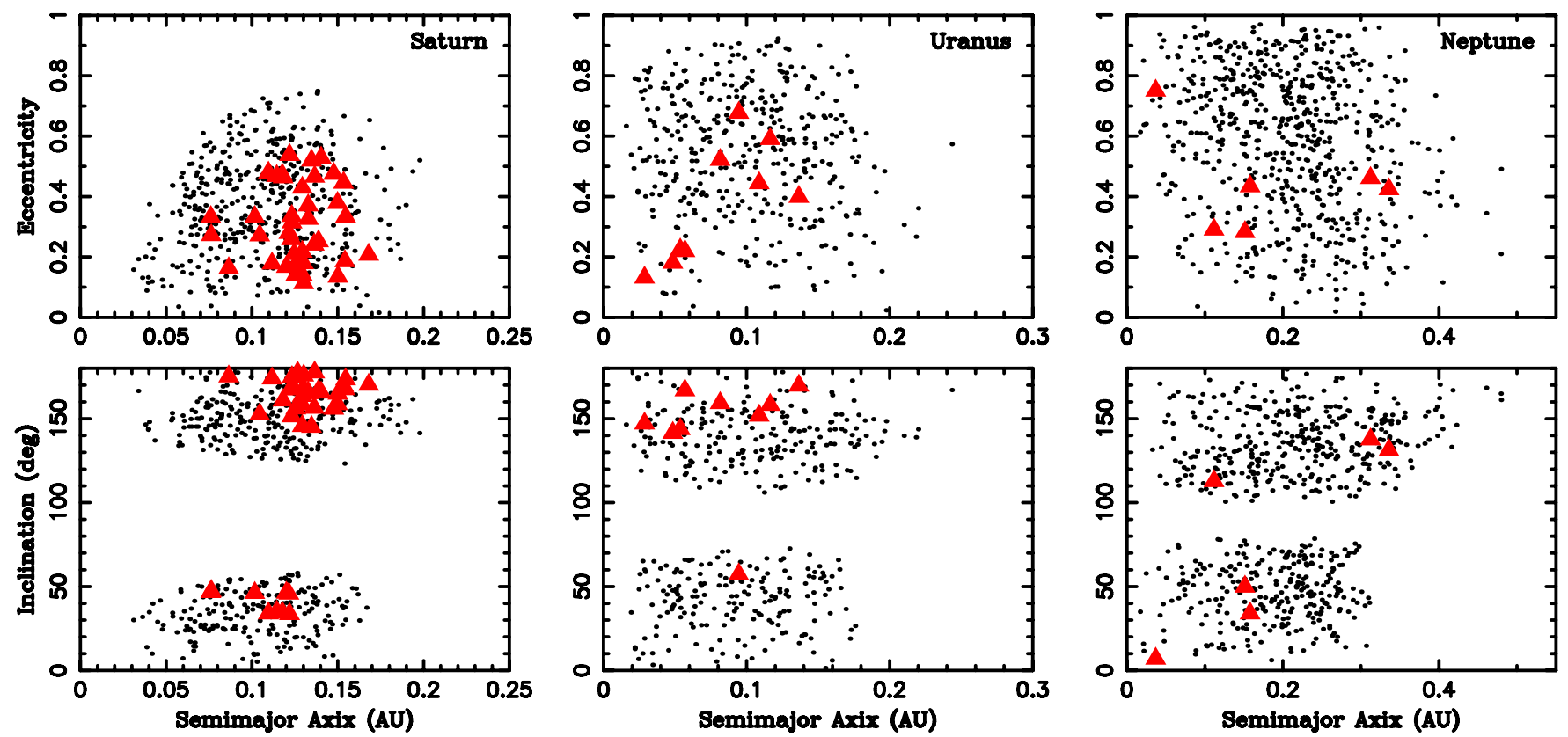

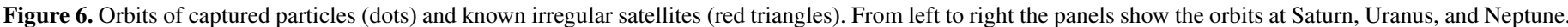
The results for Case 1 are shown here. The orbital distributions obtained in Cases 2 and 3 are similar.

(A color version of this figure is available in the online journal.)

captured at these planets. Additional differences are caused by the number and radial distribution of planetesimals at the onset of planetary encounters.

A closer investigation into this issue goes beyond the scope of this paper, because it will require exploration of a wider range of the planetary instability models. In particular, we will need to identify the initial conditions such that not only all planets have encounters during the instability, but also that the encounters occur in exactly the right proportion. ${ }^{7}$ This effort will help to better constrain the initial state from which the solar system evolved.

\section{CONCLUSIONS}

We find that planetary encounters can lead to capture of a population of irregular satellites at Jupiter that favorably compares, both in number and orbital distribution, with the known irregular moons of Jupiter. This resolves the problem identified in NVM07, where it was pointed out that the ONM does not provide a unified framework for capture of irregular satellites at all outer planets, because Jupiter does not typically have encounters with another planet. Using the jumping-Jupiter models from NM12, instead, appears to be a step in good direction, because these models allow us to extend the capture process suggested in NVM07 to Jupiter as well. Moreover, the capture probabilities obtained here for different planets are similar (to within a factor of few) explaining why the populations of irregular moons at different planets are roughly similar in number (Jewitt \& Haghighipour 2007). In broader context, the work presented here provides support for the jumping-Jupiter model (Morbidelli et al. 2009a, 2010; Brasser et al. 2009), and shows a good consistency of the planetary instability simulations published in NM12.

This work was supported by NASA's Outer Planet Research program. The work of D.V. was partly supported by the Czech

\footnotetext{
7 Note that the simulations reported here were CPU expensive such that we were unable to more fully explore parameter space.
}

Grant Agency (grant P209-13-013085). R.D. was supported by FAPESP (grants 2012/23732 and 2010/11109).

\section{REFERENCES}

Agnor, C. B., \& Lin, D. N. C. 2012, ApJ, 745, 143

Batygin, K., Brown, M. E., \& Betts, H. 2012, ApJL, 744, L3

Bottke, W. F., Nesvorný, D., Vokrouhlický, D., \& Morbidelli, A. 2010, AJ, 139, 994

Brasser, R., Morbidelli, A., Gomes, R., Tsiganis, K., \& Levison, H. F. 2009, A\&A, 507, 1053

Brasser, R., Walsh, K. J., \& Nesvorný, D. 2013, MNRAS, 433, 3417

Carruba, V., Burns, J. A., Nicholson, P. D., \& Gladman, B. J. 2002, Icar, 158,434

Colombo, G., \& Franklin, F. A. 1971, Icar, 15, 186

Ćuk, M., \& Burns, J. A. 2004, Icar, 167, 369

Duncan, M. J., Levison, H. F., \& Lee, M. H. 1998, AJ, 116, 2067

Fraser, W. C., Brown, M. E., Morbidelli, A., Parker, A., \& Batygin, K. 2014 AJ, submitted (arXiv:1401.2157)

Heppenheimer, T. A., \& Porco, C. 1977, Icar, 30, 385

Jewitt, D., \& Haghighipour, N. 2007, ARA\&A, 45, 261

Levison, H. F., \& Duncan, M. J. 1994, Icar, 108, 18

Masset, F., \& Snellgrove, M. 2001, MNRAS, 320, L55

Minton, D. A., \& Malhotra, R. 2009, Natur, 457, 1109

Morbidelli, A., Brasser, R., Gomes, R., Levison, H. F., \& Tsiganis, K. 2010, AJ, 140,1391

Morbidelli, A., Brasser, R., Tsiganis, K., Gomes, R., \& Levison, H. F. 2009a, A\&A, 507, 1041

Morbidelli, A., Levison, H. F., Bottke, W. F., Dones, L., \& Nesvorný, D. 2009b, Icar, 202, 310

Nesvorný, D. 2011, ApJL, 742, L22

Nesvorný, D., Alvarellos, J. L. A., Dones, L., \& Levison, H. F. 2003, AJ, 126,398

Nesvorný, D., \& Morbidelli, A. 2012, AJ, 144, 117 (NM12)

Nesvorný, D., Vokrouhlický, D., \& Morbidelli, A. 2007, AJ, 133, 1962 (NVM07)

Nesvorný, D., Vokrouhlický, D., \& Morbidelli, A. 2013, ApJ, 768, 45

Nicholson, P. D., Ćuk, M., Sheppard, S. S., Nesvorný, D., \& Johnson, T. V. 2008, in The Solar System Beyond Neptune, ed. M. A. Barucci et al. (Tucson, AZ: Univ. Arizona Press), 411

Philpott, C. M., Hamilton, D. P., \& Agnor, C. B. 2010, Icar, 208, 824

Pollack, J. B., Burns, J. A., \& Tauber, M. E. 1979, Icar, 37, 587

Porco, C. C., West, R. A., McEwen, A., et al. 2003, Sci, 299, 1541

Suetsugu, R., \& Ohtsuki, K. 2013, MNRAS, 431, 1709

Tsiganis, K., Gomes, R., Morbidelli, A., \& Levison, H. F. 2005, Natur, 435, 459 